\title{
A challenging pacemaker implantation in the presence of a giant right coronary aneurysm compressing the right atrium
}

\author{
Stefano Maffè, Paola Paffoni, Luca Bergamasco, Eleonora Prenna, Giulia Careri, Emanuela Facchini, \\ Lara Baduena, Nicolò Franchetti Pardo, Pierfranco Dellavesa
}

Division of Cardiology, SS Trinità Borgomanero Hospital, ASL NO, Novara, Italy

\begin{abstract}
Giant coronary artery aneurysm is an uncommon disease, treated with surgical intervention or percutaneous coil embolization. A thrombosed aneurysm can cause extrinsic compression on the cardiac chambers, with potential hemodynamic effects and may cause problems when we need to implant a cardiac device. We present a case of difficult pacemaker implantation in a patient with 3 syncopes, first-degree AV block and complete left bundle branch block on electrocardiogram. The patient presented a giant aneurysm of the right coronary artery $(85$ x $90 \mathrm{~mm})$, thrombosed, with right atrial compression. The pacemaker
\end{abstract}

Correspondence: Dr. Stefano Maffè, Division of Cardiology, SS Trinità Hospital, viale Zoppis 10, 28021 Borgomanero (NO), Italy. Tel. +39.0322.848295.

E-mail: stemaffe@libero.it

Key words: Pacemaker implantation; giant coronary aneurysm; echocardiography.

Contributions: All the authors made a substantive contribution, have read and approved the final version of the manuscript and agreed to be accountable for all aspects of the work.

Ethics approval and consent to participate: No ethical committee approval was required for this case report by the Department, because this article does not contain any studies with human participants or animals.

Consent for publication: The patient gave his written consent to use his personal data for the publication of this case report and any accompanying images.

Conflict of interest: The authors declare that they have no competing interests, and all authors confirm accuracy.

Received for publication: 12 August 2021.

Accepted for publication: 13 September 2021.

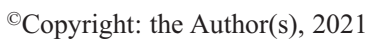

Licensee PAGEPress, Italy

Monaldi Archives for Chest Disease 2022; 92:2048

doi: 10.4081/monaldi.2021.2048

This article is distributed under the terms of the Creative Commons Attribution Noncommercial License (by-nc 4.0) which permits any noncommercial use, distribution, and reproduction in any medium, provided the original author(s) and source are credited. implantation was hampered by the difficulty of passing the lead through the compressed right atrium; indeed, only with simultaneous echocardiographic and fluoroscopic guidance, was it possible to complete the procedure. This case demonstrates the utility of echocardiogram, in particular settings, in cardiac stimulation procedures.

\section{Introduction}

Coronary artery aneurysm (CAA) is an uncommon disease, being observed in only $0.15-4.9 \%$ of patients undergoing coronary angiography [1]. CAA are defined as dilated coronary artery sections more than 1.5 times larger than the diameter of normal adjacent segments or of the patient's largest coronary vessel. CAA are considered 'giant' when they exceed $20 \mathrm{~mm}$ in diameter or 4 times the diameter of a normal coronary artery. Giant CAA are even rarer with a reported incident of $0.02-0.2 \%$ [2]. Treatment is not standardized, is individually tailored to the location and morphology of the CAA, and clinical presentation, and may include medical therapy, surgical intervention or percutaneous treatment with coil embolization $[3,4]$. A thrombosed aneurysm can cause extrinsic compression on the cardiac chambers, with potential hemodynamic effects and may cause problems when we need to implant a cardiac device. We present a case of difficult pacemaker implantation in a patient with a giant aneurysm of the right coronary artery, that determined major right atrial compression and which had previously been treated with coil embolization.

\section{Case Report}

We report the case of an 86-year-old, hypertensive, dyslipidemic, non-insulin-dependent diabetic man, with a history of chronic ischemic cardiomyopathy. In 1995 he had suffered an acute myocardial infarction and had undergone subsequent double coronary artery bypass grafting (left internal mammary artery on anterior descending artery and venous graft on branch marginal of the circumflex coronary artery). In 2013, he presented with heart failure and severe mitral insufficiency, with an indication for cardiac surgery. Pre-surgical coronary angiography revealed threevessel coronary artery disease, left internal mammary artery patency, saphenous vein graft occlusion and an aneurysm in the mid right coronary artery $(65 \times 75 \mathrm{~mm})$. The aneurysm was replete with blood, partially thrombosed and in close contact with the posterior sternal wall; the right coronary artery was distally occluded. After a collegial discussion with cardiac surgeons, the 
decision was taken to exclude the aneurysm from the circulation by means of embolization with coils, in order to avoid any damage and consequent bleeding during the initial stages of cardiac surgery (sternotomy and isolation of the pericardium). Subsequently, elective surgical replacement of the mitral valve with a biological prosthesis was performed, without complications.

In 2019 a control chest CT scan was performed, while the patient was totally asymptomatic; the right coronary aneurysm appeared enlarged ( $85 \times 90 \mathrm{~mm})$, completely thrombosed and not replete with blood, with compression of the right atrium and inferior vena cava. A conservative approach was maintained.

The patient was hospitalized in May 2021 following 3 syncopes over 20 days, with cranial trauma. On admission, he was clinically stable, had no fever, a soft abdomen without hepatomegaly, no peripheral edema, and no jugular turgor, and was normotensive $(110 / 60 \mathrm{mmHg})$. His respiratory rate was 22 breaths/min and oxygen saturation was $95 \%$. An electrocardiogram showed sinus rhythm with first-degree AV block and complete left bundle branch block. No tachy or bradyarrhythmias were recorded during hospitalization and monitoring. Chest CT confirmed the presence of the aneurysm, the dimensions of which were unchanged from 2019, with right atrial compression (Figure $1 \mathrm{a}-\mathrm{d}$ ), and without evidence of pulmonary embolism. Transthoracic and transesophageal echocardiography confirmed the compression of the mass on the right atrium, which was reduced to a very small cavity (Figure $2 \mathrm{a}, \mathrm{b}$ ). We performed a stress echocardiogram with dobutamine to evaluate a possible hemodynamic effect of atrial compression in stress conditions. However, while the HR reached $135 \mathrm{bpm}$, we did not register changes in the diastolic right and left ventricular filling pattern, significant arrhythmias, blood pressure drops or significant symptoms. On the basis of these assessments, the patient's repeated syncopes and electrocardiographic changes (with first degree AV block and complete left bundle branch block), we decided to implant a VVIR ventricular pacemaker. The procedure was hampered by the difficulty of passing the lead through the compressed right atrium in order to reach the tricuspid plane and then enter the right ventricle; indeed, only after numerous attempts, and with the aid of simultaneous echocardiographic and fluoroscopic guidance, was it possible to complete the procedure without complications (Figure 3). The patient was discharged, after a cardiac surgery evaluation, during which it was decided that a conservative approach should be maintained.

\section{Discussion}

Coronary artery aneurysms are rare findings and may be detected on coronary angiography or computed tomography. Potential complications include in situ thrombosis with distal embolization leading to myocardial infarction, local compression of adjacent structure (pulmonary artery, tricuspid valve, etc.), and possible rupture leading to sudden death due to massive tamponade [5].
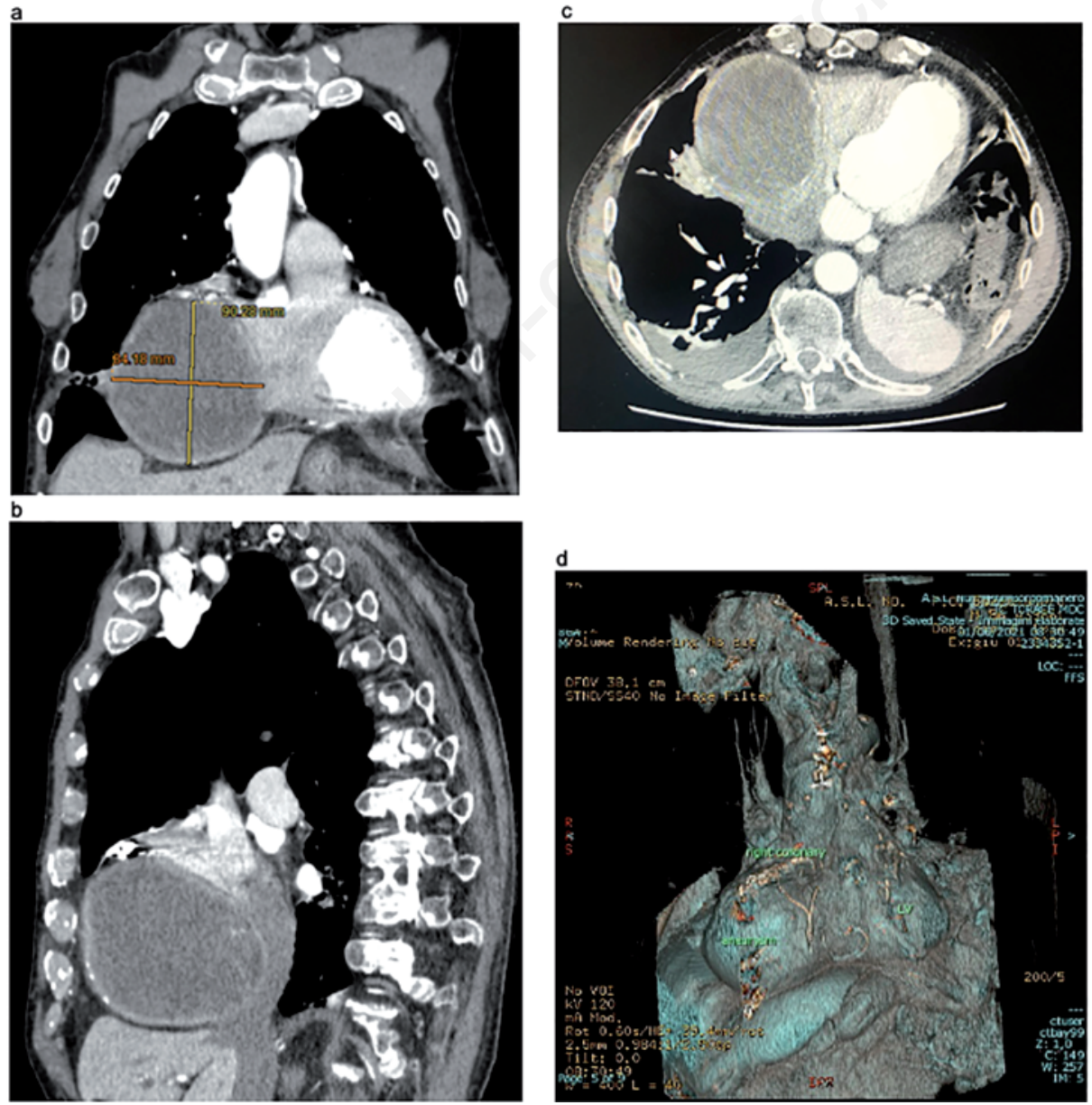

Figure 1. Chest CT confirmed the presence of the right coronary artery aneurysm $(85 \times 90 \mathrm{~mm})$, with right atrial compression. Coronal view (a); sagittal view (b); axial view (c); threedimensional CT rendering $($ d). 
The pathophysiological mechanism of the formation of coronary aneurysms is not completely understood; the most accredited hypothesis seems to be a decrease in the elastic elements of the arterial wall, which, in association with intraluminal pressure, leads to an increase in wall stress [6]. In most cases, the diagnosis of coronary aneurysms is incidental and consequent to a diagnostic coronary examination.

This case presented several interesting clinical aspects. We were unsure whether the syncopal episodes were related to conduction disturbances or extrinsic compression. However, the aneurysm was already present, and of the same size, in 2019, but the patient suffered syncopal episodes only in the 20 days prior to hospitalization. In addition, right atrial compression, even in the presence of right atrial masses, is often asymptomatic or causes symptoms such as fatigue, dyspnea, hepatomegaly, peripheral edema and jugular turgor [7]; our patient had none of these symptoms, which, in any case, are unlikely to cause syncope. We also performed a functional test under pharmacological stress and at high heart rates, to check for signs of obstruction in the right ventricular inflow; this test yielded a negative result. We therefore attributed syncope to a conduction disturbance and, consequently, implanted a permanent pacemaker. The implantation procedure itself was unusual, as we found it very difficult to pass the lead through the right atrium; we were able to position the lead in the right ventricular apex only by using simultaneous fluoroscopic and echocardiographic guidance, and adopting a substernal epigastric echocardiographic approach.

Previous studies have reported the feasibility of using intracardiac echocardiography (ICE) to guide pacemaker implantation [8]. However, ICE is expensive and requires singleuse probes. In addition, it carries a high risk of peripheral vascular complications. Thus, ICE is not widely used in current clinical practice. Transthoracic echocardiographic guidance is also little used, as it depends on the acoustic window and requires precautions in order to maintain the sterility of the operating field. However, some experiences of pacemaker implantations under exclusive ultrasound guidance, without fluoroscopy, have been reported [9]; this approach is beneficial for both patients and operators, in particular clinical settings, such as in our case. To our knowledge, this is the first report of pacemaker implantation in these conditions of extrinsic right atrial compression.

Another interesting aspect concerns the long-term evolution of embolized coronary aneurysms. Giant coronary aneurysms are extremely rare and anecdotal [10], and their natural history remains

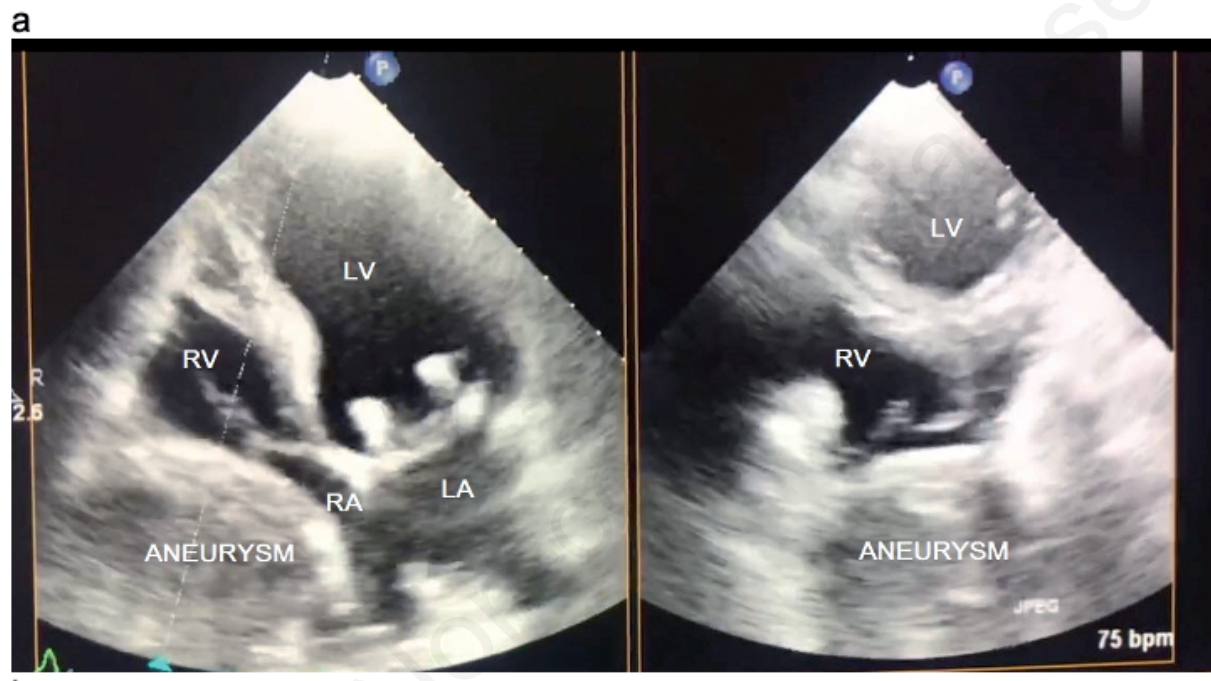

b

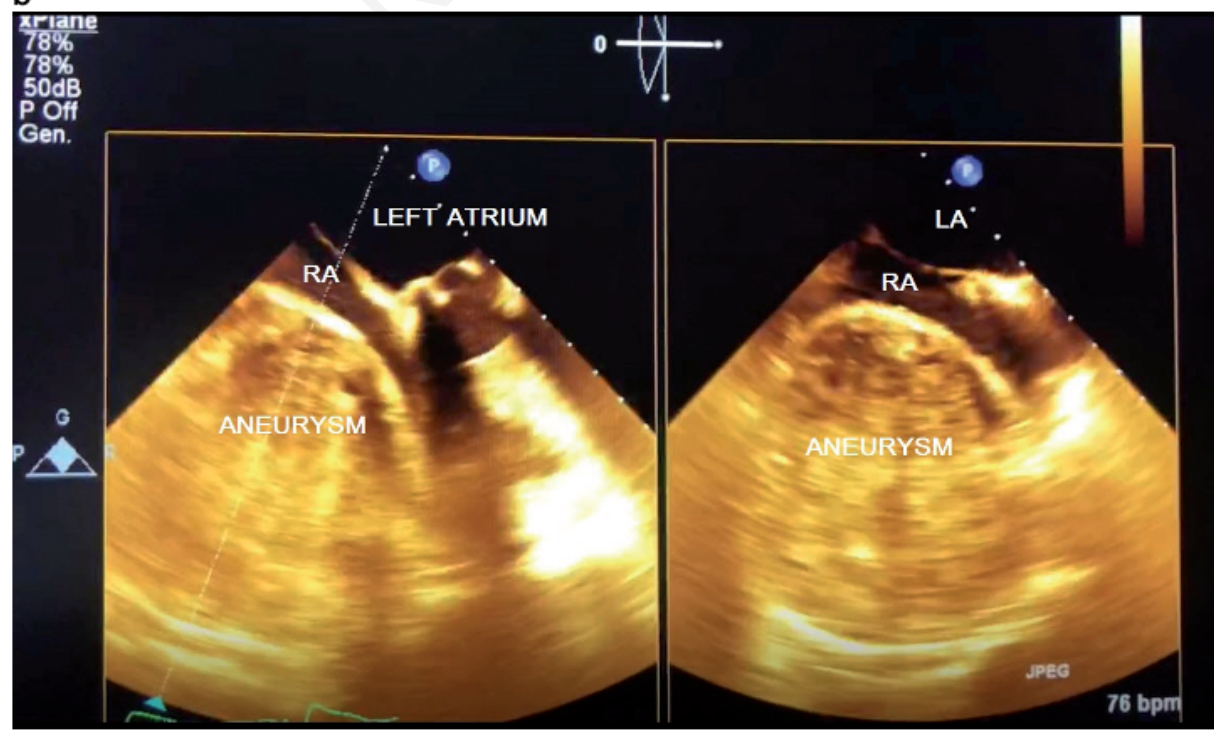

Figure 2. Transthoracic (a) and transesophageal (b) X-plane echocardiograms confirmed the compression of the mass on the right atrium, which was reduced to a very small cavity. 


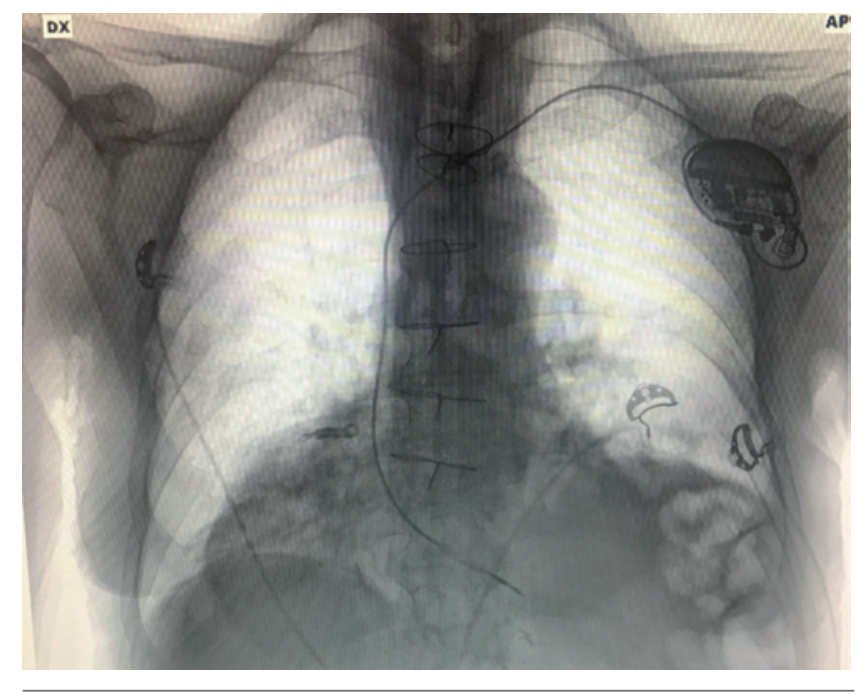

Figure 3. Postero-anterior chest X-ray after pacemaker implantation with lead positioned in the right ventricular apex.

obscure; the 5-year survival of affected patients is reported to be $71 \%$ [11] and there are no guidelines on their management. Embolization to exclude the aneurysm from the circulation is an option [4], but, as this case report shows, the long term persistence of a completely thrombosed and compact aneurysm can determine marked compression of the cardiac chambers, with potentially deleterious hemodynamic effects.

Cardiac compression caused by coronary aneurysms can cause serious effects, such as cardiogenic shock, heart failure, or arrhythmias, especially when left cardiac sections or the right ventricle are involved [12-14]. In our case, the compression, although marked, was limited to the right atrium, allowing the patient to remain asymptomatic for a long time.

How to manage aneurysms remains debatable. We opted for a conservative approach, given the patient's advanced age, the high risks involved in a possible third surgical operation and the documented absence of clear hemodynamic effects on provocative testing. Our policy was to maintain close follow-up and to implement the surgical option only in the case of clinical or hemodynamic worsening.

\section{Conclusions}

This clinical case presents some learning points: coronary aneurysms are rare but can reach important dimensions and are difficult to manage. If not surgically removed, they can create compression on the cardiac chambers. Closure with embolization is a valid therapeutic option but does not eliminate the mass effect of the aneurysm. This can be a problem in case of lead implantation, with difficulty in positioning and progression in the compressed cardiac chambers. Ultrasound guidance with a subcostal approach can support fluoroscopic guidance in these complicated procedures.

\section{References}

1. Tuncer E, Onsel Turk U, Alioglu E. Giant saccular aneurysm of the left main coronary artery. J Geriatr Cardiol 2013;10:110-2.

2. Kawsara A, Núñez Gil IJ, Alqahtani F, et al. Management of coronary artery aneurysms. JACC Cardiovasc Interv 2018;11: 1211-23.

3. Banerjee P, Houghton T, Walters M, Kaye GC. Giant right coronary artery aneurysm presenting as a mediastinal mass. Heart 2004;90:e50.

4. Rognoni A, Degiovanni A, Cavallino C F, et al. Giant aneurysm of the right coronary artery: an unusual treatment . G Ital Cardiol 2015;16:250-3.

5. Luo Y, Tang J, Liu X, Qiu J F, et al. Coronary artery aneurysm differs from coronary artery ectasia: angiographic characteristics and cardiovascular risk factor analysis in patients referred for coronary angiography. Angiology 2017;68:823-30.

6. Demopoulos VP, Olympios CD, Fakiolas CN, et al. The natural history of aneurysmal coronary artery disease. Heart 1997;78:136-41.

7. Endo A, Ohtahara A, Kinugawa T, et al. Characteristics of cardiac myxoma with constitutional signs: multicenter study in Japan. ClinCardiol 2002;25:367-70.

8. Vijayaraman P, Panikkath R. Intracardiac echocardiographyguided left bundle branch pacing in a patient with tricuspid valve replacement. J Cardiovasc Electrophysio. 2019;30:2525-7.

9. Tan K, Deng X, Wang S, et al. Transthoracic echocardiographyguided left bundle branch pacing without fluoroscopic guidance: A case report. J Clin Ultrasound 2021;49:74-7.

10. Alioglu E, Turk UO, Engin C, et al. Left main coronary artery aneurysm in young patient with acute myocardial infarction. $\mathrm{J}$ Cardiovasc Med 2009;10:494-6.

11. Halapas A, Lausberg H, Gehrig T, et al. Giant right coronary artery aneurysm in an adult male patient with non-ST myocardial infarction. Hellenic J Cardiol 2013;54:69-76.

12. Ng CY, Stevens S, Buch M, et al. Giant saphenous vein graft aneurysm causing left atrial compression and cardiogenic shock. Rev Cardiovasc Med 2010;11:e170-5.

13. Hudzik B, Glowacki J, Szkodzinski J, et al. Giant saphenous vein graft pseudoaneurysm compressing the right atrium and right ventricle and presenting as decompensated heart failure. Can J Cardiol 2011;27:390.e9-11.

14. Krishnasamy P, Gillespie JS, McGlinchey PG, et al. Giant true saphenous vein graft aneurysm causing cardiac compression: a rare cause of atrial flutter. Int J Cardiol 2007;121:317-9. 